\title{
PSYCHOGENIC NON EPILEPTIC SEIZURE STATUS - DIAGNOSTIC AND TREATMENT CHALLENGE
}

\author{
Silvio Basic ${ }^{1,2}$, Ivana Markovic ${ }^{1,2}$, Davor Sporis ${ }^{1,2}$, Ivana Susak Sporis ${ }^{1,2}$ \& Igor Filipcic ${ }^{1,3,4}$ \\ ${ }^{1}$ Josip Juraj Strossmayer University of Osijek, Faculty of Medicine, Osijek, Croatia \\ ${ }^{2}$ Department of Neurology, University Hospital Dubrava, Zagreb, Croatia \\ ${ }^{3}$ Psychiatric Hospital „Sveti Ivan“, Zagreb, Croatia \\ ${ }^{4}$ School of Medicine, University of Zagreb, Zagreb, Croatia
}

received: 26.9.2016;

revised: 1.12.2016;

accepted: 15.2 .2017

\section{INTRODUCTION}

Psychogenic non epileptic seizure status (PNES status) is defined as a recurrent or continuous seizure of psychogenic origin that may resemble those of epileptic status (ES) as there may be tongue biting, incontinence, cyanosis and even extensor plantar response (Howell et al. 1989). Minimum duration of PNES which fulfills criteria for PNES status is at least 30 minutes (Pakalnis et al. 2000). PNES status is estimated to be as frequent as epileptic status and occurs predominately in women accounting for 7183\% of all PNES statuses, or in ratio female: male 8:1 (Ramani et. al 1980, Krumholz \& Niedermeyer 1983). It occurs in about one third of patients with diagnosed psychogenic non epileptic seizures and it is frequently recurrent (Howell et al. 1989). It is a rare cause of emergency patient admission, but despite of that it represents a huge diagnostic and treatment problem. PNES status could be first presentation of underlying psychiatric disorder, it could present in patients with previously diagnosed psychogenic non epileptic seizures (PNES) or in patients with previously diagnosed epilepsy as well. In most patients PNES status could be only manifestation of underlined subconscious psychic distress or psychological disorder. Most patients do not produce symptoms consciously, except in rare cases of factitious disorder when symptoms are produced on purpose. In the assessment of patients with PNES status or epileptic status, it is very important to detect signs and symptoms which could lead to the right diagnosis. The presence of asynchronous limb shaking, closed eyes with resistance to passive opening and changes in the intensity of limb and body movements are some of determining signs indicative for PNES status. Beside clinical presentation, the gold standard in differentiation and detection of PNES and hence PNES status is video EEG monitoring.

We describe two patients who presented with PNES status which were admitted to our Centre as epileptic status.

\section{CASE 1}

A 29-year-old female patient was referred to our Centre due to epileptic status which started with a tonic spasm of the right hand followed by secondary generalization and lasted for 45 minutes. After the initial exam in the emergency room and failure to interrupt the status with parenteral administration of antiepileptic drugs according to the guidelines for the treatment of ES (Trinka et al. 2015). She was sedated, intubated, put on artificial ventilation and admitted to intensive care unit. Her initial laboratory findings (complete blood count, biochemistry, coagulation panel) were all within reference range. Thyroid function tests and prolactin level were also within reference range. The next day the patient was awakened and was transferred to our epilepsy monitoring unit (EMU) in good condition. Upon admission to the EMU long term video EEG monitoring was initiated and we observed PNES status which presented with initial bizarre spasm of the right hand followed by asynchronous, arrhythmic motoric activity of extremities in a semi flexed position with closed eyes and active resistance to passive opening of eyelids, without epileptiform EEG discharges. After thirty-five minutes status was terminated by tactile stimulation and suggestive talking to the patient. During her stay detailed medical history was revealed. The patient was a drug addict from the age of 13 to 19 (including frequent heroin abuse). She experienced one generalized epileptic seizure during the abstinence crisis and after the ingestion of a huge amount of alcohol and drugs. Six months prior to admission to our hospital she was diagnosed focal epilepsy after experiencing one dialeptic seizure with secondary generalization and carbamazepine was introduced in therapy. According to her medical records she had been experiencing one to two seizures per month. These seizures were described as long in duration (20-30 minutes), would begin with staring, followed by falling down with jerks of all extremities. During these episodes her eyes were closed and there was no postictal confusion observed. 
The frequency of seizures increased to one per week after returning to homeland from a drug addicts' commune abroad (which happened one month prior to admission to our hospital). During her stay we performed psychological testing, brain MRI (1.5T) and interictal EEG. Psychological testing revealed emotional immaturity, impulsivity and low self-control while brain MRI and interictal EEG were both normal. The patient was referred to a psychiatrist for further treatment. During consecutive year patient did not experience any seizures (neither epileptic, nor psychogenic) hence antiepileptic therapy was stopped and until now, two years after, she is still seizure free.

\section{CASE 2}

A 40-year-old female patient was referred to our hospital with the diagnosis of epileptic status. After failure of initial drug treatment in the emergency room setting, she was sedated and intubated and transferred to intensive care unit. All her laboratory findings including prolactin level were within normal range. When the patient awoke she again experienced status lasting for 35 minutes. It clinically presented as low amplitude whole body tremor with hands fisted and eyes closed. She was sedated again for the next two days. When patient awoke for second time she was transferred to our EMU where detailed history was taken. The patient reported that she had been experiencing epileptic seizures for several months. She described her seizures as an initial epigastric sensations progressing to the back, followed by partial loss of consciousness (she can sometimes hear what is going on around her) and feeling of tonic spasm of her hands after regaining consciousness. Two weeks before admission to our hospital the frequency of seizures increased up to 15 per day. Prior to the admission to our hospital she had been admitted to another clinical hospital due to epileptic status for five times. Each time she was admitted to intensive care unit due to prolonged (on average 45 minutes), asynchronous jerking of her extremities with closed eyes and each time she was sedated (using intravenous application of fentanyl, thiopental and midazolam), intubated and ventilated for several days. During her last admission the status was interrupted with sublingual application of olanzapine. In her medical record it was noted that during the last several years she had been experiencing polymorphic problems (headaches, diffuse paresthesias, vertigo, fatigue etc.) with all findings within reference range. She was diagnosed depression and anxious disorder with dominant somatic equivalent. During her stay in EMU we observed six PNES statuses which lasted from $30-$ 45 minutes with asymmetrically whole body shaking; closed eyes (with active resistance while we tried to open them) and no correlates in the electroencephalogram. We managed to terminate seizures by tactile and subtle pain stimuli (compression on mandibula or sternum, hand or leg pinching) and verbal suggestion (the need for transferring her in on psychiatry ward, the fact we knew she was reluctant to). Her 1.5T MRI and interictal EEG were normal. Psychological testing revealed depressive and anxious personality keen on hypochondriac reactions. The patient was referred to a psychiatrist and afterwards a history of domestic violence with sexual abuse was revealed. During one and a half year of her follow up she did not experience any seizures.

\section{DISCUSSION}

Although there are many described features which can help in the distinction between PNES status and ES there is still no single clinical sign which allows definite differentiation between PNES and ES. In acute clinical setting distinction can be more difficult, especially to untrained medical staff or medical staff not familiar with these conditions. This often leads to unnecessary performance of laboratory, electrophysiological and imaging studies and hence to spending extensive amounts of time and resources. Early diagnosis of PNES status is crucial to avoid inadequate and potentially harmful patient management such as parenteral administration of antiepileptic drugs, and even more important, general anesthesia, intubation and artificial ventilation due to refraction to initial therapy. Moreover, treating patients with PNES status in intensive care unit setting induces their further somatization, hence is not uncommon that those patients are subjects of multiple intensive care unit admissions. Importance of early adequate diagnosis is noted in several reported case reports on PNES status. For example, Reuber et al. described the case of a young man with borderline and factitious disorder who experienced cardiorespiratory arrest after he was introduced in general anesthesia due to PNES status resembling epileptic status (Reuber at al. 2004). There are several clinical signs which can help in distinction between PNES status and ES. During PNES status clonic muscle jerks are often asymmetrical, asynchronous, with variable intensity and frequently present pelvic thrusting. Eyes are often closed with voluntary rejection when tried to be open by examiner and classic postictal confusion is absent. The concomitant occurrence with epileptic seizures often complicates detection and treatment of PNES and PNES status. The stigma of having a psychiatric disorder and its denial can make a therapeutic alliance with patients experiencing PNES status problematic. Different types of cognitive behavioral therapy are treatment modalities are preferred in those patients (Bodde et al. 2009). Better treatment outcome is associated with younger age at presentation, female sex and different seizure types. The success of treatment deteriorates during time so the early identification of patients with PNES/PNES status is very important for a good treatment outcome (Gudmundsson et al. 2008). 


\section{CONCLUSION}

According to our experience the acute treatment of PNES status should be focused on tactile, pain and verbal stimuli (dominantly concerning patient's management which the patient is reluctant to). After successful acute management of PNES status, it is obligatory to refer the patient to a psychiatrist since it is known that early psychiatric management is crucial in the treatment of patients with PNES seizures and PNES status as well.

\section{Acknowledgements: None.}

Conflict of interest: None to declare.

\section{Contribution of individual authors:}

Silvio Basic was involved with manuscript preparation and review of draft manuscript.

Ivana Markovic was involved with patient data collection, manuscript preparation.

Ivana Susak Sporis was involved with literature searches, manuscript preparation.

Davor Sporis was involved with manuscript preparation, literature searches.

Igor Filipcic reviewed draft manuscript.

\section{References}

1. Bodde NM, Brooks JL, Baker GA, Boon PA, Hendriksen JG, Mulder OG, Aldenkamp AP: Psychogenic nonepileptic seizures-definition, etiology, treatment and prognostic issues: a critical review. Seizure 2009; 18:543-53

2. Gudmundsson O, Prendergast $M$, Foreman D, Cowley S: Outcome of pseudoseizures in children and adolescents: $a$ 6-year symptom survival analysis. Dev Med Child Neurol 2001; 43:547-51.

3. Howell SJ, Owen L, Chadwick DW: Pseudostatus epilepticus. Q J Med 1989; 71:507-19.

4. Krumholz A, Niedermeyer E: Psychogenic seizures: a clinical study with follow-up data. Neurology 1983; 33:498-502.

5. Pakalnis A, Paolicchi J, Gilles E: Psychogenic status epilepticus in children: psychiatric and other risk factors. Neurology 2000; 54:969-70.

6. Ramani SV, Quesney LF, Olson D, Gumnit RJ: Diagnosis of hysterical seizures in epileptic patients. Am J Psychiatry 1980; 137:705-9.

7. Reuber M, Baker GA, Gill R, Smith DF, Chadwick DW: Failure to recognize psychogenic nonepileptic seizures may cause death. Neurology 2004; 62:834-5.

8. Trinka E, Höfler J, Leitinger M, Brigo F: Pharmacotherapy for Status Epilepticus. Drugs 2015; 75:1499-521.

\section{Correspondence:}

Asst. Prof. Silvio Basic, MD, PhD

Department of Neurology, University Hospital Dubrava Av. Goika Suska 6, 10000 Zagreb, Croatia

E-mail: sbasic@mefos.hr; sbasic@kbd.hr 